\title{
Non-abelian anomalies and transport *
}

\author{
Juan L. Mañes ${ }^{1, * *}$, Eugenio Megías ${ }^{2,3, * * *}$, Manuel Valle ${ }^{3, * * *}$, and Miguel Á. Vázquez- \\ $\mathrm{Mozo}^{4, \dagger}$ \\ ${ }^{1}$ Departamento de Física de la Materia Condensada, Universidad del País Vasco UPV/EHU, Apartado \\ 644, 48080 Bilbao, Spain \\ ${ }^{2}$ Departamento de Física Atómica, Molecular y Nuclear and Instituto Carlos I de Física Teórica y Com- \\ putacional, Universidad de Granada, Avenida de Fuente Nueva s/n, 18071 Granada, Spain \\ ${ }^{3}$ Departamento de Física Teórica, Universidad del País Vasco UPV/EHU, Apartado 644, 48080 Bilbao, \\ Spain \\ ${ }^{4}$ Departamento de Física Fundamental, Universidad de Salamanca, Plaza de la Merced s/n, 37008 Sala- \\ manca, Spain
}

\begin{abstract}
We study the role of quantum anomalies in relativistic fluids of nonabelian theories within the hydrodynamic expansion. To this end, we compute the local functional that solves the anomaly equations, and obtain the BardeenZumino terms that covariantize the currents. We particularize these results to a background with an electromagnetic field and chiral imbalance for two flavors.
\end{abstract}

\section{Introduction}

Hydrodynamics is an effective description of out-of equilibrium systems in which local thermodynamical equilibrium is assumed [1]. The equations of motion correspond to the conservation laws of the energy-momentum tensor and charge currents, which are supplemented by expressions of these quantities written in terms of fluids variables, the so-called constitutive relations. In presence of anomalies the currents are no longer conserved, and this has important effects in the hydrodynamics. In the general case, in addition to the ideal hydrodynamical contributions, there are extra terms in the constitutive relations which lead to dissipative and anomalous effects, i.e. for the charge currents $\left\langle J^{\mu}\right\rangle=n u^{\mu}+\left\langle J^{\mu}\right\rangle_{\text {diss \& anom. Two relevant }}$ phenomena appear at first order in the hydrodynamic expansion as a consequence of chiral anomalies: the chiral magnetic effect, which is responsible for the generation of an electric current induced by an external magnetic field in the fluid $B^{\mu}=\varepsilon^{\mu v \rho \lambda} u_{v} \partial_{\rho} \mathbb{V}_{\lambda}$ [2], and the chiral vortical effect, in which the electric current is induced by a vortex $\omega^{\mu}=\varepsilon^{\mu v \rho \lambda} u_{v} \partial_{\rho} u_{\lambda}$ [3], i.e. $\left\langle J^{\mu}\right\rangle_{\text {anom }}=\sigma^{\mathscr{B}} B^{\mu}+\sigma^{\mathscr{V}} \omega^{\mu}+\cdots$. The corresponding susceptibilities $\sigma^{\mathscr{B}}$ and $\sigma^{\mathscr{V}}$ are even under time reversal symmetry, so that they cannot contribute to entropy production, i.e. $\partial_{t} s_{\text {anom }}=0$, and then they lead to non-dissipative effects [4]. Recently it has been proposed a convenient formalism to obtain the non-dissipative part of the anomalous constitutive relations, which is based on the existence of an equilibrium partition function in a stationary

\footnotetext{
*Talk given by E. Megías at the QCD@Work: International Workshop on QCD, 25-28 June 2018, Matera, Italy.

**e-mail: wmpmapaj@lg.ehu.es

***e-mail: emegias@ugr.es

****e-mail: manuel.valle@ehu.es

†e-mail: Miguel.Vazquez-Mozo@cern.ch
} 
background $[5,6]$. The dissipative effects like, for instance, the shear viscosity or the electric conductivity, demand the use of alternative methods, e.g. kinetic theory, Kubo formulae or fluid/gravity correspondence [7-9]. In this work we will apply the equilibrium partition function formalism to study the anomalous part of the constitutive relations in non-abelian theories.

\section{Equilibrium partition function formalism and hydrodymamics}

We present in this section a summary of the equilibrium partition function formalism introduced in $[5,6]$ (see also e.g. $[10,11]$ ). Let us consider a relativistic invariant Quantum Field Theory with a time independent $\mathrm{U}(1)$ gauge connection on the manifold ${ }^{1}$

$$
\begin{aligned}
d s^{2} & =G_{\mu v} d x^{\mu} d x^{v}=-e^{2 \sigma(\vec{x})}\left(d t+a_{i}(\vec{x}) d x^{i}\right)^{2}+g_{i j}(\vec{x}) d x^{i} d x^{j}, \\
\mathscr{A} & =\mathscr{A}_{0}(\vec{x}) d x^{0}+\mathscr{A}_{i}(\vec{x}) d x^{i} .
\end{aligned}
$$

As usual, the partition function of the system writes

$$
Z=\operatorname{Tr} e^{-\frac{H-\mu_{0} Q}{T_{0}}},
$$

where $H$ is the Hamiltonian of the theory, and $Q$ is the charge associated to the gauge connection. $T_{0}$ and $\mu_{0}$ are the temperature and chemical potential at equilibrium. The most general partition function should be consistent with: i) 3-dim diffeomorphism invariance; ii) Kaluza-Klein (KK) invariance, i.e. $t \rightarrow t+\phi(\vec{x}), \vec{x} \rightarrow \vec{x}$; and iii) $\mathrm{U}(1)$ time-independent gauge invariance (up to an anomaly).

From the partition function we can compute the energy-momentum tensor and $\mathrm{U}(1)$ charged current by performing the appropriate $t$-independent variations, i.e.

$$
\delta \log Z=\frac{1}{T_{0}} \int d^{3} x \sqrt{g_{3}}\left(-\frac{1}{2} T_{\mu \nu} \delta g^{\mu v}+J^{\mu} \delta \mathscr{A}_{\mu}\right),
$$

where $g_{3}=\operatorname{det}\left(g_{i j}\right)$. In particular, for a general dependence $\log Z=$ $\mathscr{W}\left(e^{\sigma}, A_{0}, a_{i}, A_{i}, g^{i j}, T_{0}, \mu_{0}\right)$, where

$$
A_{0}=\mathscr{A}_{0}, \quad A_{i}=\mathscr{A}_{i}-a_{i} \mathscr{A}_{0},
$$

are KK invariant quantities, one gets the consistent currents and energy-momentum tensor

$$
\begin{aligned}
\left\langle J^{i}\right\rangle_{\text {cons }} & =\frac{T_{0}}{\sqrt{-G}} \frac{\delta \mathscr{W}}{\delta A_{i}}, & \left\langle J_{0}\right\rangle_{\text {cons }} & =-\frac{T_{0} e^{2 \sigma}}{\sqrt{-G}} \frac{\delta \mathscr{W}}{\delta A_{0}}, \\
\left\langle T_{0}^{i}\right\rangle & =\frac{T_{0}}{\sqrt{-G}}\left(\frac{\delta \mathscr{W}}{\delta a_{i}}-A_{0} \frac{\delta \mathscr{W}}{\delta A_{i}}\right), & \left\langle T_{00}\right\rangle & =-\frac{T_{0} e^{2 \sigma}}{\sqrt{-G}} \frac{\delta \mathscr{W}}{\delta \sigma} .
\end{aligned}
$$

It is clear from these expressions that $\mathscr{W}$ plays the role of a generating functional for the hydrodynamic constitutive relations.

Let us discuss now the properties of the partition function at first order in the hydrodynamic expansion. The most general form of the partition function in this case, compatible with the symmetries mentioned above, is [5, 11]:

$$
\mathscr{W}^{(1)}=\int d^{3} x \sqrt{g_{3}}\left[\alpha_{1}(T, v) \varepsilon^{i j k} A_{i} A_{j k}+\alpha_{2}(T, v) \varepsilon^{i j k} A_{i} f_{j k}+\alpha_{3}(T, v) \varepsilon^{i j k} a_{i} f_{j k}\right],
$$

\footnotetext{
${ }^{1}$ For simplicity, we will be restricted to abelian theories in this section. However, we will generalize this analysis to the non-abelian case in Sec. 3.
} 
where

$$
T=e^{-\sigma} T_{0}, \quad v=\frac{A_{0}}{T_{0}},
$$

while $A_{i j}=\partial_{i} A_{j}-\partial_{j} A_{i}$ and $f_{i j}=\partial_{i} a_{j}-\partial_{j} a_{i}$. The specific values of the functions $\alpha_{i}(T, v)$ depend on the particular theory considered. In practice, one way to compute $\alpha_{i}$ is to insert Eq. (7) into (6), and compare the result with the constitutive relations for that theory. In particular, if one considers an ideal gas of Dirac fermions, a suitable computation of the constitutive relations leads to the following expressions for the chiral magnetic and vortical conductivities

$$
\sigma^{\mathscr{B}}=C \mu, \quad \sigma^{\mathscr{V}}=\frac{1}{2} C \mu^{2}+C_{2} T^{2} \mu
$$

where we have used that $v=\mu / T$ with $\mu=e^{-\sigma} A_{0}$. These results have been obtained in a wide variety of methods, see e.g. [2, 3, 9, 11-19]. In these expressions $C=1 /\left(4 \pi^{2}\right)$ and $C_{2}=$ $1 / 24$ are related to the axial anomaly $[3,20]$ and mixed gauge-gravitational anomaly [13], respectively. ${ }^{2}$ From this, the result for the partition function in this theory turns out to be

$$
\alpha_{1}(T, v)=-\frac{C}{6} v, \quad \alpha_{2}(T, v)=-\frac{1}{2}\left(\frac{C}{6} v^{2}-C_{2}\right), \quad \alpha_{3}(T, v)=0 .
$$

In the following we will operate in the opposite way, i.e. starting from the partition function, we will compute the constitutive relations of the theory. $\mathscr{W}$ will be obtained by solving the anomaly equations.

\section{Non-abelian anomalies}

In this section we will study the partition function in non-abelian theories. Our goal is to obtain from it the constitutive relations. We refer to [21,22] for full details in the computation.

\subsection{The chiral anomaly}

We begin by studying the theory of a chiral fermion coupled to an external gauge field $\mathscr{A}_{\mu}^{a}$ described by the Lagrangian

$$
\mathscr{L}_{\mathrm{YM}}=i \bar{\psi} \gamma^{\mu}\left(\partial_{\mu}-i t_{a} \mathscr{A}_{\mu}^{a}\right) \psi
$$

where $t_{a}=t_{a}^{\dagger}$ are the Hermitian generators of the Lie algebra satisfying the commutation relations $\left[t_{a}, t_{b}\right]=i f_{a b c} t_{c}$. To study gauge anomalies it is convenient to work with the fermion effective action functional obtained by integrating out the fermion field

$$
e^{i \Gamma[\mathscr{A}]} \equiv \int \mathscr{D} \bar{\psi} \mathscr{D} \psi e^{i S_{\mathrm{YM}}[\mathscr{A}, \psi, \bar{\psi}]} .
$$

Under a general shift $\mathscr{A}_{\mu}^{a} \rightarrow \mathscr{A}_{\mu}^{a}+\delta \mathscr{A}_{\mu}^{a}$, one gets that the first order variation of $\Gamma[\mathscr{A}]$ can be expressed as

$$
\delta \Gamma[\mathscr{A}]=\int d^{D} x \delta \mathscr{A}_{\mu}^{a}(x) J_{a}^{\mu}(x)_{\mathrm{cons}}
$$

\footnotetext{
${ }^{2}$ If we have a set of chiral fermions transforming under a Lie group generated by $t_{a}$ in the background of arbitrary gauge fields and metric, then the axial and mixed gauge-gravitational anomalies are proportional to $\sim \operatorname{Tr}\left(t_{a}\left\{t_{b}, t_{c}\right\}\right)$ and $\sim \operatorname{Tr}\left(t_{a}\right)$, respectively.
} 
where $J_{a}^{\mu}(x)_{\text {cons }}$ is the consistent current. The axial anomaly is given by the failure of the effective action to be invariant under axial gauge transformations, which are defined as

$$
\mathscr{A}_{\mu} \longrightarrow U \mathscr{A}_{\mu} U^{-1}-i \partial_{\mu} U U^{-1}, \quad U(x)=\exp \left(i \Lambda_{a}^{\mathrm{A}}(x) t_{a}\right)
$$

Under such a transformation,

$$
\delta_{\text {gauge }} \Gamma[\mathscr{A}]=-\int d^{D} x \Lambda_{a}^{\mathrm{A}}(x) G_{a}[\mathscr{A}(x)]
$$

where $G_{a}[\mathscr{A}(x)]$ is the consistent anomaly. Particularizing now Eq. (13) to a gauge transformation $\delta \mathscr{A}_{\mu}^{a}=\left(D_{\mu} \Lambda^{\mathrm{A}}\right)^{a}$, we arrive at the anomalous (non)-conservation law for the consistent current

$$
D_{\mu} J_{a}^{\mu}(x)_{\mathrm{cons}}=G_{a}[\mathscr{A}(x)] .
$$

We will analyze in the following the consequences of these results for the hydrodynamics of fluids affected by gauge anomalies.

\subsection{Non-abelian anomaly and anomalous functional}

In the following we will consider a non-abelian theory with symmetry group $\mathrm{U}\left(N_{f}\right) \times \mathrm{U}\left(N_{f}\right)$. The Bardeen form of the anomaly in this theory is [23]

$$
\begin{aligned}
G_{a}[\mathscr{V}, \mathscr{A}]= & \frac{i N_{c}}{16 \pi^{2}} \varepsilon^{\mu v \rho \sigma} \operatorname{Tr}\left\{t _ { a } \left[\mathscr{V}_{\mu v} \mathscr{V}_{\rho \sigma}+\frac{1}{3} \mathscr{A}_{\mu v} \mathscr{A}_{\rho \sigma}-\frac{32}{3} \mathscr{A}_{\mu} \mathscr{A}_{v} \mathscr{A}_{\rho} \mathscr{A}_{\sigma}\right.\right. \\
& \left.\left.+\frac{8}{3} i\left(\mathscr{A}_{\mu} \mathscr{A}_{v} \mathscr{V}_{\rho \sigma}+\mathscr{A}_{\mu} \mathscr{V}_{\rho \sigma} \mathscr{A}_{v}+\mathscr{V}_{\rho \sigma} \mathscr{A}_{\mu} \mathscr{A}_{v}\right)\right]\right\}
\end{aligned}
$$

where $t_{a}$ are the generators of the Lie algebra of $\mathrm{U}\left(N_{f}\right)$, while

$$
\begin{aligned}
\mathscr{V}_{\mu v} & =\partial_{\mu} \mathscr{V}_{v}-\partial_{v} \mathscr{V}_{\mu}-i\left[\mathscr{V}_{\mu}, \mathscr{V}_{v}\right]-i\left[\mathscr{A}_{\mu}, \mathscr{A}_{v}\right] \\
\mathscr{A}_{\mu v} & =\partial_{\mu} \mathscr{A}_{v}-\partial_{v} \mathscr{A}_{\mu}-i\left[\mathscr{V}_{\mu}, \mathscr{A}_{v}\right]-i\left[\mathscr{A}_{\mu}, \mathscr{V}_{v}\right]
\end{aligned}
$$

are the field strengths for the vector and axial gauge fields. Notice that the anomaly includes not only triangle, but also square and pentagon one-loop diagram contributions.

Our first goal is to compute the effective action compatible with the anomaly (17). The anomaly arises from the breaking of gauge invariance under axial gauge transformations of the effective action $\Gamma[\mathscr{V}, \mathscr{A}]$, so that such an action should satisfy

$$
\mathscr{Y}_{a}(x) \Gamma[\mathscr{V}, \mathscr{A}]=0, \quad \mathscr{X}_{a}(x) \Gamma[\mathscr{V}, \mathscr{A}]=G_{a}[\mathscr{V}, \mathscr{A}]
$$

where $\mathscr{Y}_{a}(x)$ and $\mathscr{X}_{a}(x)$ are the local generators of vector and axial gauge transformations, respectively. Then the computation of the local functional $\Gamma[\mathscr{V}, \mathscr{A}]$ can be performed, for instance, by solving Eq. (19) by trial and error. The details of this computation will be presented in [22], and we will just show here the final result, which reads

$$
\begin{aligned}
\Gamma[V, A]= & -\frac{N_{c}}{32 \pi^{2}} \int d t d^{3} x \sqrt{g_{3}} \varepsilon^{i j k} \operatorname{Tr}\left\{\frac{32}{3} i V_{0} A_{i} A_{j} A_{k}\right. \\
& +\frac{4}{3}\left(A_{0} A_{i}+A_{i} A_{0}\right) A_{j k}+4\left(V_{0} A_{i}+A_{i} V_{0}\right) V_{j k} \\
& \left.+\frac{8}{3}\left(A_{0}^{2}+3 V_{0}^{2}\right) A_{i} \partial_{j} a_{k}\right\}+C_{2} T_{0}^{2} \int d t d^{3} x \sqrt{g_{3}} \varepsilon^{i j k} \operatorname{Tr} A_{i} \partial_{j} a_{k},
\end{aligned}
$$


where $V_{\mu}$ and $A_{\mu}$ are KK invariant vector and axial gauge fields, cf. Eq. (5). Let us mention at this point that $\Gamma$ can be determined also from differential geometry methods, i.e. starting from the anomaly polynomial, one can compute the Chern-Simons effective action and to obtain from there the $t$-independent anomalous effective action after a suitable dimensional reduction, see e.g. [21, 24] for details. As it has been mentioned in Sec. 2, the coefficient $C_{2}$ is related to the mixed gauge-gravitational anomaly $\sim \operatorname{Tr}\left(t_{a}\right) \varepsilon^{\mu \nu \rho \sigma} R_{\mu \nu \lambda \kappa} R_{\rho \sigma}{ }^{\lambda \kappa}$. Following similar differential geometry methods, it would be possible to compute this contribution by taking into account the Riemann tensor effect in the anomaly polynomial $\sim \operatorname{Tr} \mathscr{F}_{A} R^{\mu}{ }_{v} R_{\mu}^{v}$. However, in the following we will neglect these contributions as this analysis goes beyond the scope of the present work. ${ }^{3}$

\subsection{Covariant currents and constitutive relations}

The charged currents obtained from the functional derivatives of the effective action are consistent currents, cf. Eq. (6). However, only covariant currents can enter in the constitutive relations, and these are defined by adding to the consistent currents the Bardeen-Zumino (BZ) polynomials, i.e. ${ }^{4}$

$$
J_{\mathrm{cov}}^{\mu}=J_{\mathrm{cons}}^{\mu}+J_{\mathrm{BZ}}^{\mu} .
$$

The BZ polynomials for this theory have been computed in [21, 22], leading to the following result

$$
\begin{aligned}
& J_{\mathrm{BZV}}^{\mu}=\frac{N_{c}}{8 \pi^{2}} \varepsilon^{\mu v \rho \sigma} \operatorname{Tr}\left\{t_{a}\left(\mathscr{A}_{v} \mathscr{V}_{\rho \sigma}+\mathscr{V}_{\rho \sigma} \mathscr{A}_{v}+\frac{8}{3} i \mathscr{A}_{v} \mathscr{A}_{\rho} \mathscr{A}_{\sigma}\right)\right\}, \\
& J_{\mathrm{BZA}}^{\mu}=\frac{N_{c}}{24 \pi^{2}} \varepsilon^{\mu v \rho \sigma} \operatorname{Tr}\left\{t_{a}\left(\mathscr{A}_{v} \mathscr{A}_{\rho \sigma}+\mathscr{A}_{\rho \sigma} \mathscr{A}_{v}\right)\right\} .
\end{aligned}
$$

Then the covariant currents and stress tensor in equilibrium can be obtained from $\mathscr{W}=i \Gamma$, and using Eqs. (21) and (22). This procedure leads in the vector currents to contributions of the form [22]

$$
\left\langle J_{\mathrm{V} a}^{i}\right\rangle_{\operatorname{cov}} \propto e^{\sigma} \varepsilon^{i j k} \operatorname{Tr}\left\{t_{a} A_{0} V_{j k}\right\} \quad \text { and } \quad\left\langle J_{\mathrm{V} a}^{i}\right\rangle_{\operatorname{cov}} \propto e^{\sigma} \varepsilon^{i j k} \operatorname{Tr}\left\{t_{a} A_{0} V_{0} \partial_{j} a_{k}\right\},
$$

which remind the chiral magnetic and vortical effects, respectively. ${ }^{5}$ Similar contributions appear in the axial current $\left\langle J_{\mathrm{A} a}^{i}\right\rangle_{\operatorname{cov}}$ and energy-momentum tensor. It is interesting to note that the lower temporal indices, $\left\langle J_{\mathrm{V} a 0}\right\rangle_{\mathrm{cov}}$ and $\left\langle J_{\mathrm{A} a 0}\right\rangle_{\mathrm{cov}}$, as well as the components of the energy-momentum tensor, $\left\langle T_{00}\right\rangle$ and $\left\langle T^{i j}\right\rangle$, are vanishing.

While these results are valid for a non-abelian theory with symmetry group $\mathrm{U}\left(N_{f}\right) \times$ $\mathrm{U}\left(N_{f}\right)$, it would be interesting to particularize them for a specific background. It is widely accepted that, in presence of non-abelian charges, the maximal number of chemical potentials to be consistently introduced corresponds to the dimension of the Cartan subalgebra. Then, for $N_{f}=2$, we can consider the background given by a combination of $t_{0}=\frac{1}{2} 1_{2 \times 2}$ and $t_{3}=\frac{1}{2} \sigma_{3}$, where $\sigma_{i}$ are the Pauli matrices, i.e.

$$
V_{\mu}(\boldsymbol{x})=V_{0 \mu}(\vec{x}) t_{0}+V_{3 \mu}(\vec{x}) t_{3}, \quad A_{0}=A_{50} t_{0}, \quad A_{i}=0 .
$$

For simplicity, we assume that $A_{50}$ is constant. Then, in addition to the equilibrium velocity field $u_{\mu}=-e^{\sigma}(1, \vec{a})$, we can define the equilibrium baryonic, isospin and axial chemical potentials, as

$$
\mu_{0}=\mathscr{V}_{00} e^{-\sigma}, \quad \mu_{3}=\mathscr{V}_{30} e^{-\sigma}, \quad \mu_{5}=\mathscr{A}_{50} e^{-\sigma},
$$

\footnotetext{
${ }^{3}$ See [25] for an alternative analysis of these contributions in a group theoretic formulation.

${ }^{4}$ In fact, it is not possible to define an effective action whose derivation with respect to the gauge field gives the covariant current [15].

${ }^{5} \mathrm{~A}$ reliable identification of each term of (23) with a specific chiral effect can only be done after a computation of the out-of-equilibrium currents in a particular background, as it is done below.
} 
where $\mu_{5}$ controls the chiral imbalance of the system. In heavy ion collisions, the chiral imbalance is induced by a difference between the numbers of left-handed and right-handed quarks in the fireball [26, 27]. Accordingly, we can distinguish between three currents. For Quantum Chromodynamics (QCD) with $u$ and $d$ quarks, with electric charges $+\frac{2}{3} e$ and $-\frac{1}{3} e$, respectively, these are

$$
\begin{aligned}
J_{\mathrm{V}, \text { electromagnetic }}^{\mu} & =\frac{e}{3} \bar{\psi} \gamma^{\mu} t_{0} \psi+e \bar{\psi} \gamma^{\mu} t_{3} \psi, \\
J_{\mathrm{V}, \text { baryonic }}^{\mu} & =\frac{2}{3} e \bar{\psi} \gamma^{\mu} t_{0} \psi \\
J_{\mathrm{V}, \text { isospin }}^{\mu} & =e \bar{\psi} \gamma^{\mu} t_{3} \psi
\end{aligned}
$$

in the vector sector, which obviously fulfill the Gell-Mann-Nishijima formula. Finally, the out-of-equilibrium expressions of the currents follow from a Lorentz covariantization of the equilibrium currents (23). We will present here only partial results, and leave the detailed analysis together with the complete results for [22]. In particular, for the baryonic current one finds

$$
\begin{aligned}
& \left\langle J_{\mathrm{V}, \text { baryonic }}^{\mu}\right\rangle_{\mathrm{cov}}=-\frac{N_{c} e^{2}}{72 \pi^{2}} \mu_{5} \varepsilon^{\mu v \lambda \rho} u_{v} \mathbb{V}_{\lambda \rho}, \\
& \left\langle J_{\mathrm{A}, \text { baryonic }}^{\mu}\right\rangle_{\mathrm{cov}}=\frac{N_{c} e}{24 \pi^{2}}\left(\mu_{5}^{2}-\mu_{0}^{2}-\mu_{3}^{2}\right) \varepsilon^{\mu \nu \lambda \rho} u_{v} \partial_{\lambda} u_{\rho}+\cdots,
\end{aligned}
$$

in the vector and axial sector, respectively. We have used that $\mathscr{V}_{0 \mu}=\frac{e}{3} \mathbb{V}_{\mu}$ and $\mathscr{V}_{3 \mu}=e \mathbb{V}_{\mu}$, where $\mathbb{V}_{\mu}$ is the electromagnetic field and $\mathbb{V}_{\mu \nu}=\partial_{\mu} \mathbb{V}_{v}-\partial_{v} \mathbb{V}_{\mu}$ its field strength. We can easily identify in Eq. (27) the chiral magnetic and vortical contributions in the baryonic current, as well as the explicit results for the corresponding conductivities. Similar results can be obtained for the other currents and for the energy-momentum tensor. ${ }^{6}$

\section{Conclusions}

In this work we have studied non-dissipative transport effects of non-abelian theories up to first order in the hydrodynamic expansion. In particular, we have analyzed the effects induced by external electromagnetic fields and vortices in a relativistic fluid. The computation has been performed by using the equilibrium partition function formalism which, by construction, is suitable for the study of time reversal properties, i.e. those effects that cannot lead to entropy production. The local functional relevant for the computation of the constitutive relations has been obtained by solving the anomaly equations. Finally, we have found the results of the constitutive relations for the covariant currents after adding the corresponding Bardeen-Zumino polynomials. It would be interesting to extend this study to the case of spontaneous symmetry breaking, as this would lead to relevant information about the hydrodynamics of the corresponding Goldstone bosons interacting with external fields, with an obvious application to QCD at low temperatures. These and other issues will be addressed in a forthcoming publication [22].

\section{Acknowledgement}

This work has been supported by Plan Nacional de Altas Energías Spanish MINECO grants FPA201564041-C2-1-P, FPA2015-64041-C2-2-P, and by Basque Government grant IT979-16. The research of E.M. is also supported by Spanish MINEICO and European FEDER funds grant FIS2017-85053-C21-P, Junta de Andalucía grant FQM-225, as well as by Spanish MINEICO Ramón y Cajal Program and

\footnotetext{
${ }^{6}$ See e.g. [28] for a related study of chiral anomalies in superfluids.
} 
by Universidad del País Vasco UPV/EHU, Bilbao, Spain, through a Visiting Professor appointment. M.A.V.-M. gratefully acknowledges the hospitality of the KEK Theory Center and the Department of Theoretical Physics of the University of the Basque Country during the early stages of this work.

\section{References}

[1] P. Kovtun, J. Phys. A45, 473001 (2012).

[2] K. Fukushima, D.E. Kharzeev, H.J. Warringa, Phys. Rev. D78, 074033 (2008).

[3] D.T. Son, P. Surowka, Phys. Rev. Lett. 103, 191601 (2009).

[4] D.E. Kharzeev, H.U. Yee, Phys. Rev. D84, 045025 (2011).

[5] N. Banerjee, J. Bhattacharya, S. Bhattacharyya, S. Jain, S. Minwalla, T. Sharma, JHEP 09, 046 (2012).

[6] K. Jensen, P. Kovtun, A. Ritz, JHEP 10, 186 (2013).

[7] P.B. Arnold, G.D. Moore, L.G. Yaffe, JHEP 05, 051 (2003).

[8] G. Policastro, D.T. Son, A.O. Starinets, Phys. Rev. Lett. 87, 081601 (2001).

[9] J. Erdmenger, M. Haack, M. Kaminski, A. Yarom, JHEP 01, 055 (2009).

[10] S. Bhattacharyya, JHEP 08, 165 (2014).

[11] E. Megías, M. Valle, JHEP 11, 005 (2014).

[12] N. Banerjee, J. Bhattacharya, S. Bhattacharyya, S. Dutta, R. Loganayagam, P. Surowka, JHEP 01, 094 (2011).

[13] K. Landsteiner, E. Megías, F. Pena-Benitez, Phys. Rev. Lett. 107, 021601 (2011).

[14] K. Landsteiner, E. Megías, L. Melgar, F. Pena-Benitez, JHEP 09, 121 (2011).

[15] K. Landsteiner, E. Megías, F. Pena-Benitez, Lect. Notes Phys. 871, 433 (2013).

[16] K. Jensen, R. Loganayagam, A. Yarom, JHEP 02, 088 (2013).

[17] E. Megías, K. Landsteiner, F. Pena-Benitez, Acta Phys. Polon. Supp. 6, 45 (2013).

[18] E. Megías, F. Pena-Benitez, JHEP 05, 115 (2013).

[19] K. Landsteiner, E. Megías, F. Pena-Benitez, Phys. Rev. D90, 065026 (2014).

[20] D.E. Kharzeev, H.J. Warringa, Phys. Rev. D80, 034028 (2009).

[21] J.L. Mañes, E. Megías, M. Valle, M.Á. Vázquez-Mozo (2018), 1806.07647.

[22] J.L. Mañes, E. Megías, M. Valle, M.Á. Vázquez-Mozo, work in progress (2018).

[23] W.A. Bardeen, Phys. Rev. 184, 1848 (1969).

[24] K. Jensen, R. Loganayagam, A. Yarom, JHEP 05, 110 (2014).

[25] V.P. Nair, R. Ray, S. Roy, Phys. Rev. D86, 025012 (2012).

[26] R. Gatto, M. Ruggieri, Phys. Rev. D85, 054013 (2012).

[27] X. Planells, A.A. Andrianov, V.A. Andrianov, D. Espriu, PoS QFTHEP2013, 049 (2013), 1310.4434.

[28] Y. Neiman, Y. Oz, JHEP 09, 011 (2011). 